Revue d'histoire de l'Amérique française

Q8. REVUE D.HISTOIRE DE L'AMÉRIQUE FRANÇAISE

\title{
La différenciation des producteurs laitiers et le marché de Montréal (1900-1930)
}

\section{Yves Otis}

Volume 45, numéro 1, été 1991

URI : https://id.erudit.org/iderudit/304947ar

DOI : https://doi.org/10.7202/304947ar

Aller au sommaire du numéro

\section{Éditeur(s)}

Institut d'histoire de l'Amérique française

\section{ISSN}

0035-2357 (imprimé)

1492-1383 (numérique)

Découvrir la revue

\section{Citer cet article}

Otis, Y. (1991). La différenciation des producteurs laitiers et le marché de Montréal (1900-1930). Revue d'histoire de l'Amérique française, 45(1), 39-71. https://doi.org/10.7202/304947ar

\section{Résumé de l'article}

Cet article vient étoffer le dossier de la participation des producteurs laitiers au marché montréalais du lait de consommation entre 1900 et 1930. L'étude de l'évolution des divers marchés du lait dans ces années montre l'émergence d'une spécialisation régionale des activités de vente des producteurs laitiers québécois. La conjoncture de l'entre-deux-guerres et les conditions particulières du développement du réseau de collecte et de distribution de lait de Montréal amènent ces fournisseurs à se démarquer des autres producteurs laitiers. Une distinction se crée alors entre vendeurs de lait de consommation et vendeurs de lait de transformation. Pour une part, cette division résulte de l'initiative des producteurs, par le biais des associations de producteurs spécialisés. Elle est aussi le fruit de la réglementation croissante du commerce du lait qui s'ébauche dès le milieu des années 1920. 


\title{
LA DIFFÉRENCIATION DES PRODUCTEURS LAITIERS ET LE MARCHÉ DE MONTRÉAL $(1900-1930)^{1}$
}

\author{
YVES OTIS \\ Département d'histoire \\ Université de Montréal
}

\section{RÉSUMÉ}

Cet article vient étoffer le dossier de la participation des producteurs laitiers au marché montréalais du lait de consommation entre 1900 et 1930. L'étude de l'évolution des divers marchés du lait dans ces années montre l'émergence d'une spécialisation régionale des activités de vente des producteurs laitiers québécois. La conjoncture de l'entre-deux-guerres et les conditions particulières du développement du réseau de collecte et de distribution de lait de Montréal amènent ces fournisseurs à se démarquer des autres producteurs laitiers. Une distinction se crée alors entre vendeurs de lait de consommation et vendeurs de lait de transformation. Pour une part, cette division résulte de l'initiative des producteurs, par le biais des associations de producteurs spécialisés. Elle est aussi le fruit de la réglementation croissante du commerce du lait qui s'ébauche dès le milieu des années 1920.

\section{ABSTRACT}

This article fills out our knowledge of the producers who supplied Montreal's consumer milk market in the years between 1900 and 1930. The study of the various markets for milk reveals a pattern of regional specialization among Quebec's dairy farmers. The economic conjuncture of the inter-war years and the particular conditions surrounding the development of a supply and distribution network for the Montreal market led these suppliers to distinguish themselves from other dairy farmers. A distinction was created between producers who supplied the consumer market and those who supplied milk to the butter and cheese factories. This differentiation was partly the result of producer initiative, expressed through specialized dairy farmers' associations. It was also related to increasing government regulation over the sale of milk, a trend that was taking shape in the mid 1920s.

1 Je tiens à remercier Tom Wien, Normand Séguin et Sylvie Taschereau pour leur lecture attentive et leurs commentaires. Les remarques formulées par Bettina Bradbury et ses étudiantes ont également servi à bonifier une première version de ce texte.

RHAF, vol. 45, nº 1, été 1991 
"Il s'est cassé, nous dit-on, quelques mâchoires; ils [sic] s'est renversé quelques bidons de lait; il s'est échangé quelques gros mots, par ci par là, dans la région de Montréal, entre producteurs et producteurs et distributeurs de lait de Montréal, la semaine dernière.»

J.-N. Ponton, Bulletin des Agriculteurs, 10 octobre 1929.

À la mi-septembre 1929, les grandes laiteries distributrices de Montréal et les associations de producteurs de lait se réunissent afin de fixer les conditions générales de vente pour la saison d'hiver. C'est habituellement sans heurt que ces négociations bi-annuelles se déroulent. Cette fois, les distributeurs offrent d'acheter le lait à 28 cents le gallon et de prolonger la saison d'un mois: ce faisant, ils favorisent leurs fournisseurs réguliers en maintenant un prix élevé pendant cette période de l'année où l'approvisionnement est difficile ${ }^{2}$. Une nouvelle organisation de producteurs se dissocie de l'entente et exige 35 cents le gallon. Devant le refus des compagnies laitières, les membres de l'Association des producteurs de lait de la province de Québec organisent un blocus de l'approvisionnement de lait de Montréal. A partir du 1er octobre 1929, ils interdisent l'accès aux gares et aux stations d'expédition et ils établissent des barrages sur certaines des routes menant à la ville.

Les nombreux reportages qui paraissent dans les journaux témoignent de la vivacité de l'affrontement: aux coups de poing et aux bidons renversés, évoqués par Ponton, s'ajoutent quelques coups de feu, quelques blessés, plusieurs assemblées publiques courues par les producteurs, les curés, les maires et les députés. La prompte réaction du gouvernement provincial, soit l'intervention directe du premier ministre Louis-Alexandre Taschereau et du ministre de l'Agriculture J.-L. Perron, indique l'ampleur du conflit. L'arbitrage du ministre Perron mène finalement à un compromis, qui comprend l'instauration d'une enquête sur les coûts de production, une réforme des lois sur les coopératives et une hausse du prix de vente du lait (32 cents) pour quatre des six mois de la saison d'hiver. Ironiquement, deux semaines plus tard, le jeudi noir marque le début d'une période de difficultés économiques qui se chargera d'annuler - provisoirement - ces modestes gains ${ }^{3}$.

2 Compte tenu des techniques d'alimentation et de la qualité du cheptel de l'époque, la production laitière est marquée par de fortes variations saisonnières: à la baisse dès la mise en stabulation des animaux pour l'hiver, à la hausse à leur sortie au printemps. Dans le cas du lait de consommation, on distingue deux grandes saisons, celle d'hiver s'échelonnant généralement d'octobre à avril. Par conséquent, les producteurs qui réussissent à étirer la période de traite sur l'année peuvent profiter du meilleur prix du lait d'hiver.

3 Dans le volume 31 de son Histoire de la province de Québec (Montréal, Fides, 1959, 31: 60-61), Robert Rumilly consacre à la grève l'équivalent d'une page, décrivant ce qui lui 
Plusieurs chercheurs ont vu dans cette grève un signe probant de l'émergence d'un type d'agriculture familiale plus décidément tourné vers le marché, qui serait confiné, pendant l'entre-deux-guerres, aux régions de Montréal et des Cantons de l'Est. Dans son étude des premières années de l'Union catholique des cultivateurs (UCC), Robert Migner présente la grève comme une confrontation entre les intermédiaires capitalistes du secteur agro-alimentaire et les cultivateurs «progressistes» de la région montréalaise ${ }^{4}$. Dans le cas présent, ces derniers tentent d'obtenir, par le biais de leurs associations professionnelles spécialisées, un meilleur contrôle des conditions de mise en marchés. De son côté, Michel Morisset reprend la présentation de Migner et l'intègre à son interprétation de l'évolution de l'agriculture familiale québécoise ${ }^{6}$; la grève des producteurs laitiers atteste selon lui l'existence d'une agriculture familiale marchande diversifiée qui ne réussira à s'imposer qu'à partir de la Seconde Guerre mondiale.

En somme, ces auteurs constatent l'existence dans la plaine montréalaise d'une agriculture familiale intégrée au marché. Leurs analyses insistent sur les conditions immédiates qui favorisent son essor, soit la Première Guerre mondiale et l'urbanisation rapide de la région. Ils ne donnent cependant que de brèves indications sur ses origines et ses assises. Pour mieux saisir la nature des transformations de l'agriculture familiale québécoise depuis le milieu du $\mathrm{XIX}^{\mathrm{e}}$ siècle, nous devons accorder davantage d'attention à la mise en place et à l'intensification

semble être une victoire habile de J.-L. Perron. Robert-M. Migner propose une tout autre interprétation dans un article paru dans Québec Presse en 1974: il y commente la victoire des producteurs organisés sur les intermédiaires capitalistes de l'agro-business ( «La grande grève agricole de 1929», Québec Presse, 22 septembre 1974, 8; voir également sa thèse, Le monde agricole québécois et les premières années de l'Union catholique des cultivateurs (1918-1930), thèse de Ph.D. (histoire), Université de Montréal, avril 1975. Dans les deux cas, les auteurs ne semblent avoir consulté que les principaux quotidiens francophones et les journaux agricoles. J'ai repris cette chronique des événements en consultant systématiquement tous les quotidiens francophones et anglophones montréalais, les hebdomadaires et les périodiques de la région touchée ainsi que les périodiques agricoles et ce, pour la période allant de la mi-septembre à la mi-octobre 1929.

4 Robert M. Migner, Le monde agricole québécois..., 276-287.

5 Le discours de l'UCC s'éloigne de plus en plus des préoccupations très concrètes de ces producteurs -- ce qui explique sa faible implantation dans la région montréalaise. Voir JeanPierre Kesteman, Histoire du syndicalisme agricole au Québec. UCC-UA, 1924-1984 (Montréal, Boréal Express, 1984), 89-90, 68 et 112. Voir également Claude Beauchamp, «Les débuts de la coopération et du syndicalisme agricoles, 1900-1930: quelques éléments de la pratique», Recherches sociographiques, 20,3 (septembre-décembre 1979): 337-381.

6 Pour la présentation du conflit, il faut consulter sa thèse, Agriculture familiale ou capitaliste au Québec au XX siècle?, thèse de doctorat d'État, Université de Paris VII (Vincennes), 1982. Son analyse du développement de l'agriculture familiale québécoise est exposée de façon plus serrée dans le livre qu'il a tiré de sa thèse, L'agriculture familiale au Québec (Paris, Éditions L'Harmattan, 1987). 
des rapports qui existent entre les producteurs agricoles et les différents marchés: celui du travail, celui de la terre, celui des denrées agricoles et celui des biens de production. Cela nous amène, entre autres, à nous interroger sur les changements qui se produisent lorsque l'exploitation agricole familiale réalise une part de plus en plus importante de la valeur de ses produits sur le marché des denrées.

En ce qui concerne le Québec, les historiens ont souligné le rôle prépondérant que les activités laitières ont joué dans cette transition ${ }^{7}$. Mettant à profit la manne de rapports, d'enquêtes, de discours et de statistiques produits sur ce sujet depuis les années 1870 , plusieurs chercheurs ont déjà établi les conditions et la chronologie du développement de ce secteur de production. Une conjonction de facteurs explique l'essor rapide de la production laitière sur les fermes québécoises à partir de ce moment: parmi les principaux, mentionnons la présence d'une demande relativement ferme de fromage sur le marché britannique, qu'accompagnent de brèves ouvertures du marché américain au beurre et à la crème du Canada, l'existence d'une production domestique qui dégage un surplus commercialisable ${ }^{8}$ et les efforts des gouvernements, tant fédéral que provinciaux, pour établir, encadrer et soutenir les réseaux de fabrication et de mise en marché. Pour dynamique qu'elle soit, cette progression a-t-elle, à terme, modifié en profondeur l'organisation et l'intensité des relations des producteurs avec le marché? La relative lenteur observée dans la transformation des fermes québécoises avant la Deuxième Guerre mondiale oblige à réévaluer l'apport du commerce d'exportation de beurre et de fromage sur ce plan ${ }^{9}$. Les faiblesses structurelles du réseau des fabriques sont bien connues: les petites installations sont nombreuses et elles produisent de façon irrégulière ou saisonnière des marchandises dont la qualité est souvent médiocre ${ }^{10}$. Cette fragilité explique peut-être que les producteurs s'accommodent des revenus de la vente de leur surplus estival de lait, sans pour autant changer de façon substantielle l'organisation de la production.

7 Jean Hamelin et Yves Roby, Histoire économique du Québec, 1850-1896 (Montréal, Fides, 1971); Normand Perron, «Genèse des activités laitières 1850-1960», N. Séguin, Agriculture et colonisation au Québec. Aspects historiques (Montréal, Boréal Express, 1980), 113-140; Normand Séguin, «L'agriculture de la Mauricie et du Québec 1850-1950», Revue d' histoire de l'Amérique française, 35,4 (mars 1982): 537-562.

8 Marjorie Cohen, «The Decline of Women in Canadian Dairying», Histoire socialel Social History, 17,34 (novembre 1984): 317-320; aussi du même auteur, Women's Work, Markets, and Economic Development in Nineteenth-Century Ontario (Toronto, University of Toronto Press, 1988), 103-106.

9 N. Séguin, «L’agriculture de la Mauricie et du Québec...», 554.

10 N. Perron, «Genèse...», 129-130 et 124. 
On pourrait reprocher à ces travaux de ne donner qu'une vue partielle des activités laitières, l'attention étant portée sur le secteur dominant de la fabrication et de l'exportation de fromage et de beurre au détriment des autres débouchés. Ainsi, les producteurs laitiers des Cantons de l'Est qui expédient leur crème aux États-Unis ou encore ceux qui approvisionnent les villes en lait de consommation ${ }^{11}$ entretiennent des contacts beaucoup plus fréquents et autrement plus intenses avec le marché. Sur la nature et l'ampleur de ces activités de vente de lait et de crème, on ne connaît que des bribes. On sait, par exemple, du commerce du lait vendu pour la consommation à Montréal les nombreux problèmes liés à la qualité du ravitaillement ${ }^{12}$, mais peu de choses sur les prix, sur le niveau de consommation, sur le réseau de distribution ou sur le bassin des fournisseurs.

Ce texte vient étoffer le dossier de la participation des producteurs laitiers au marché montréalais entre 1900 et 1930 . Je propose d'abord de suivre l'évolution des divers marchés du lait au cours de ces années, pour m'attacher ensuite aux formes que prend la participation des producteurs agricoles québécois à ces marchés au début des années 1920. Je m'intéresse plus précisément à ces nombreux exploitants agricoles qui ont choisi de vendre leur lait en exclusivité aux grandes laiteries distributrices de Montréal. Les conditions particulières du développement du réseau de collecte et de distribution de lait de cette ville amènent ces fournisseurs à se démarquer progressivement des autres producteurs laitiers. Une distinction se crée alors entre vendeurs de lait de consommation et vendeurs de lait de transformation - ou lait industriel —, distinction qui est encore vive aujourd'hui. Cette division me paraît être le résultat de l'initiative des producteurs eux-mêmes, qui tendent à se regrouper en associations spécialisées (celles-là mêmes qui sont au cœur de la grève de 1929). Elle est aussi le fruit de la réglementation du commerce du lait qui s’ébauche dès le milieu des années 1920.

11 C'est-à-dire la vente de lait et de crème pour consommation en nature. En fait, l'utilisation du lait peut être différente lorsqu'il y a surplus d'approvisionnement, comme cela se produit pendant la saison estivale. Il peut être écrémé, transformé en beurre ou en fromage. Dans ce cas, il est payé à un prix moindre par les distributeurs et les fabricants.

12 Terry Copp, Classe ouvrière et pauvreté à Montréal (Montréal, Boréal Express, 1978), 213; Martin Tétreault, "Les maladies de la misère - aspects de la santé publique à Montréal - 1880-1914», RHAF, 36,4 (mars 1983): 507-526. 


\section{LES MARCHÉS DU LAIT, 1900-1930}

Déplacée par la forte concurrence ontarienne et américaine du marché du blé, l'agriculture québécoise du milieu du $\mathrm{XIX}^{\mathrm{e}}$ siècle tente d'occuper de nouveaux créneaux sur le marché international. Le bref épisode du traité de Réciprocité et de la guerre civile américaine en avait laissé entrevoir plusieurs; l'exportation de fromage sur le marché britannique s'avère finalement le plus prometteur et le plus stable. Ce commerce connaît un essor considérable de la Confédération jusqu'au début du $\mathrm{XX}^{\mathrm{e}}$ siècle. Le Canada exporte en moyenne 15 millions de livres de fromage pour la décennie 1870-1874; pour les cinq premières années du XX $\mathrm{XX}^{\mathrm{e}}$ siècle, ce sont en moyenne 209 millions de livres de fromage qui sont écoulées sur les marchés étrangers. En fait, pour toute cette période, la quasi-totalité de la production canadienne, rarement moins de $90 \%$, est acheminée vers les consommateurs britanniques ${ }^{13}$. En 1904, point culminant de ce commerce, le fromage canadien procure $72 \%$ des importations fromagères britanniques ${ }^{14}$.

Comme le souligne Marjorie Cohen, l'expansion des activités laitières prend initialement appui sur la fabrication domestique ${ }^{15}$. Cependant, la confection de fromage sur les fermes recule rapidement, bousculée par l'implantation d'installations de transformation pouvant traiter le lait de plusieurs exploitations. Le nombre de fromageries québécoises bondit de 25 en 1871 à 1207 en 1901, essor suivi par les beurreries et les installations mixtes (combinant la fabrication de beurre et de fromage). Au tournant du siècle, ce réseau de fabriques couvre presque complètement le territoire de la province. Le commerce d'exportation qu'il supporte est animé par un nombre grandissant d'agents expéditeurs, d'acheteurs, d'inspecteurs et d'experts gouvernementaux ${ }^{16}$. Les exploitants agricoles ne sont pas en reste; encouragés par le revenu procuré par la vente de lait aux fabriques, ils augmentent progressivement la taille de leur troupeau laitier, adoptent un système de production associant les élevages laitier et porcin et consacrent plus d'espace aux productions fourragères ${ }^{17}$.

13 Raymond Duhaime, «Les exportations canadiennes de produits laitiers, 1867 à 1955», L'Actualité économique, 23,2 (juillet-septembre 1957): 247-248.

14 H. A. Innis, The Dairy Industry in Canada (Toronto, Ryerson Press, 1937), 64.

15 Marjorie G. Cohen, «The Decline of Women...», 319.

16 Il y aurait une recherche à faire sur le commerce du beurre et du fromage. Les sources ne manquent pas pour établir les conditions et l'état de ce commerce et pour dresser un portrait des agents engagés dans ces activités (fabricants, agents expéditeurs, commerçants de gros, inspecteurs, etc.).

17 Normand Séguin, «L'agriculture de la Mauricie...»; S. Courville et N. Séguin, Le monde rural québécois au 19ème siècle (Société historique du Canada, brochure historique no 47), 18-21. 
Cette croissance accélérée entraîne également son lot de problèmes, longuement discutés dans les documents. Un trop grand nombre de petites fabriques ont été créées, fonctionnant irrégulièrement et fabriquant des produits de qualité inégale, pour ne pas dire inférieure. La vie précaire de ces établissements, aux propriétaires peu argentés, occasionne souvent des pertes importantes aux patrons (fournisseurs) des fabriques ${ }^{18}$. Chez ces derniers, les spécialistes de la Société d'industrie laitière ne cessent d'observer et de déplorer le manque de soins donnés à la fois aux animaux et au lait: il en résulte une livraison irrégulière, pas toujours hygiénique, limitée à la saison estivale.

La situation du marché international des produits laitiers se transforme au tournant du $\mathrm{XX}^{\mathrm{e}}$ siècle. De nouveaux et de forts concurrents, soit la Nouvelle-Zélande et le Danemark, affaiblissent la position du fromage et du beurre canadiens sur le marché britannique. La diminution du nombre de fromageries québécoises pendant les années 19001930 traduit l'affaissement progressif du volume des exportations: nombre de ces installations disparaissent tandis que d'autres s'adaptent en entreprenant la fabrication de beurre ${ }^{19}$. Prenant la relève du marché britannique, l'essor démographique des villes nord-américaines commande un approvisionnement de plus en plus important et régulier de produits laitiers. Ces populations urbaines consomment du lait, de la crème, du beurre, mais aussi toute une gamme de «nouveaux» produits tels que la crème glacée, le lait en poudre, le lait concentré, etc. L'effort de production suscité par la Première Guerre mondiale favorisera cette première - et modeste - diversification dans l'utilisation du lait.

Durant les vingt premières années du $\mathrm{XX}^{\mathrm{e}}$ siècle, les activités de production et de transformation du lait ont consolidé leur position dans l'agriculture québécoise tout en montrant des signes nets d'essoufflement, résultat d'un développement rapide et désor-

18 Ceci dit sous toute réserve car on ne sait pas grand chose sur le mode - en particulier la régularité- de paiement des producteurs par les fabricants. Les patrons sont-ils payés au moment de la livraison du lait, à la quinzaine ou après la vente du beurre et du fromage aux agents et aux marchands? Les quelques dossiers de faillite de fromageries et de beurreries que j'ai consultés, pour les années 1920 et pour le district judiciaire de Joliette, laissent voir des dettes importantes aux fournisseurs de lait. Que sait-on finalement de la durée de vie de ces petites installations? Ce seul sujet mériterait d'être étudié avec soin, d'autant plus qu'en cours de recherche, il m'a semblé que la documentation ne manquait pas. Outre les dossiers de faillite, signalons les listes de propriétaires d'installations publiées dans plusieurs rapports de la Société d'industrie laitière, les formulaires de déclarations de formation de société; et même, avec un peu de chance, des documents de fabriques et de laiteries, tels ceux de la Crèmerie de l'Économie de Saint-Hyacinthe (ANQ, 06-M-P210, microfilm no 3711).

19 On compte 1207 fromageries en 1901, soit près de la moitié des installations de transformation laitière, et seulement 416 en 1931, moins du tiers du total des fabriques. N. Perron, "Genèse des activités laitières...», 119 et 129. 


\section{Figure 1}

\section{Fromageries, 1919-1921}

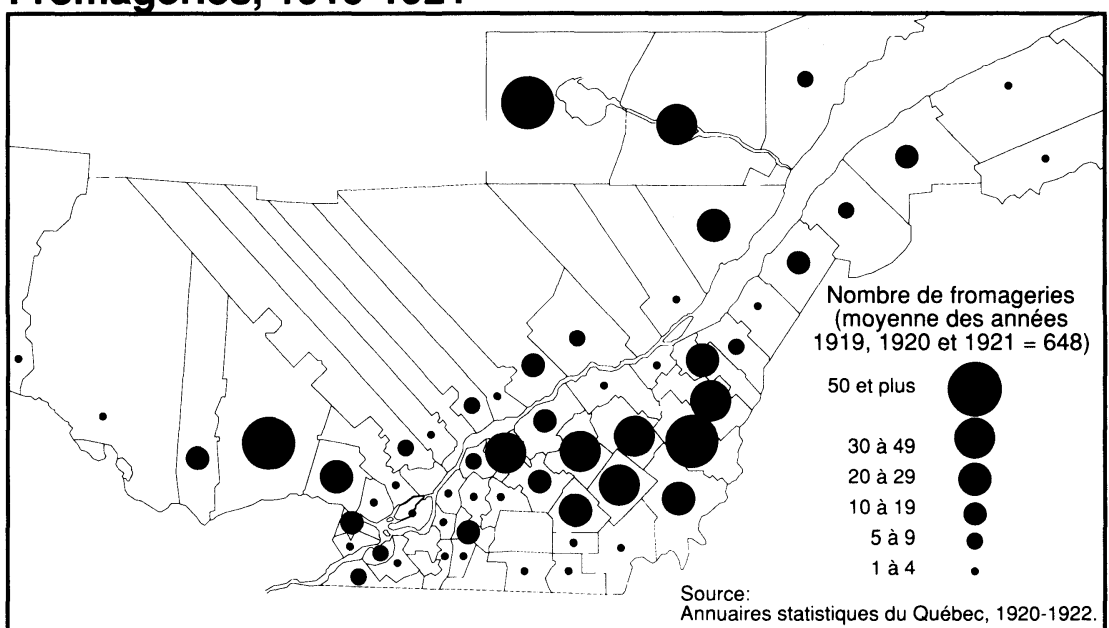

\section{Figure 2}

Beurreries, 1919-1921

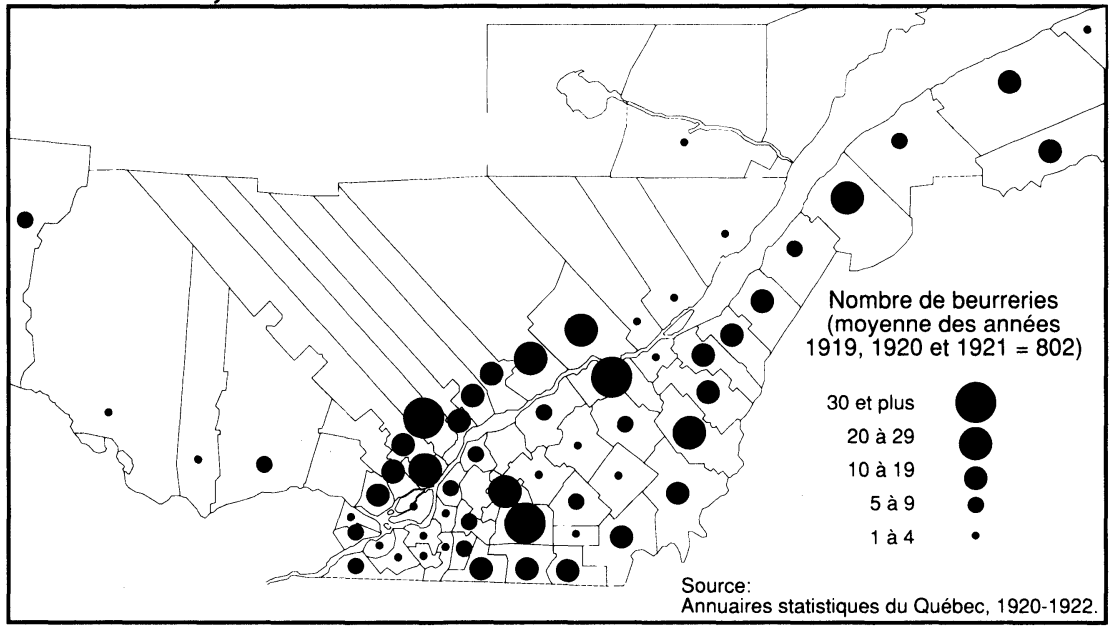




\section{Figure 3}

\section{Beurreries-fromageries, 1919-1921}

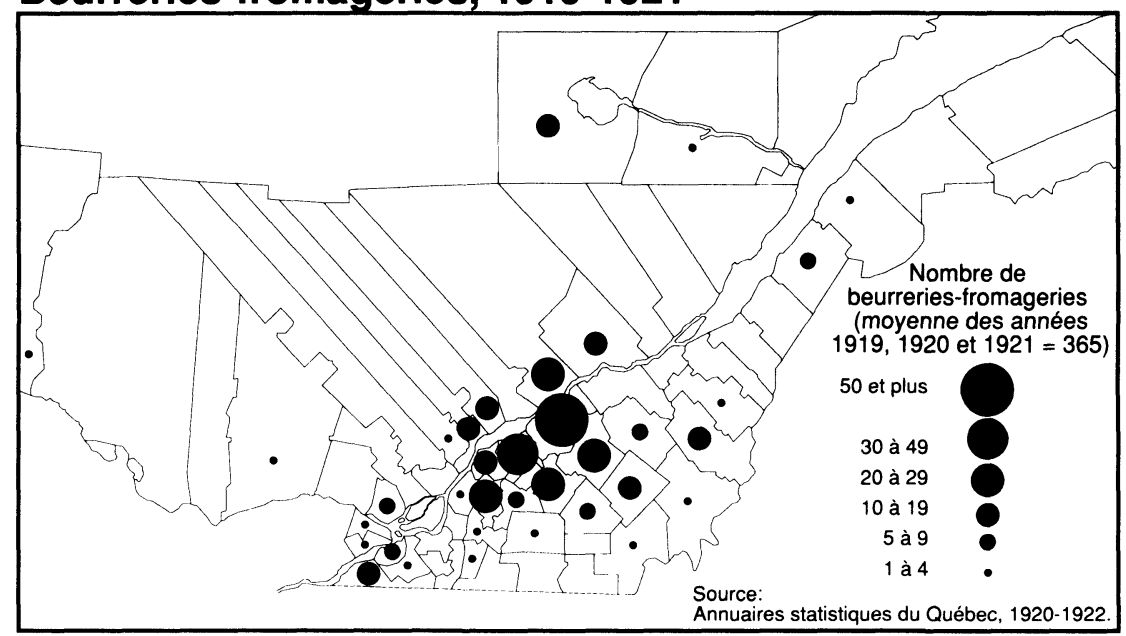

donné $^{20}$. Voilà le constat paradoxal que dresse Normand Perron, qui poursuit en qualifiant les années de l'entre-deux-guerres de période de marasme. Il est donc opportun de s'arrêter au début des années 1920 pour tenter d'évaluer la participation des producteurs laitiers aux divers débouchés.

Par son ampleur, le réseau des fabriques oriente la répartition des ventes de lait et de crème sur le territoire du Québec. Fromageries, beurreries et installations mixtes ne sont pas uniformément distribuées sur le territoire de la province (figures 1 à 3 ). Les installations fromagères dominent dans trois grandes zones (figure 1): sur la rive sud du Saint-Laurent, un croissant, partant du comté de Yamaska vers le comté de Bellechasse; dans la région du Saguenay; et, dans la zone Argenteuil-Labelle, à l'ouest de Montréal. L'implantation des beurreries s'oppose à celle des fromageries; elles sont plus nombreuses sur la rive nord du Saint-Laurent, de Deux-Montagnes à Québec, dans les Cantons de l'Est et dans le Bas-Saint-Laurent (figure 2). Quant aux fabriques combinant les deux types de production, elles font bloc au centre de la province, près du comté de Saint-Hyacinthe, siège de la Société d'industrie laitière qui est la grande promotrice de ce type d'établissements (figure 3).

Ces trois cartes font voir des régions encombrées d'installations de transformation, au centre de la province notamment, et d'autres 
zones moins bien dotées. La cartographie du rapport de fermes laitières par fabrique confirme cette inégale couverture du réseau de fabriques (figure 4) ${ }^{21}$. Le rapport utilisé masque cependant des réalités diverses. Les fronts de colonisation progressant dans la vallée de l'Outaouais et dans le Bas Saint-Laurent contribuent à affaiblir l'image de l'industrie laitière dans ces régions. Pour le Bas SaintLaurent, Bruno Jean a déjà souligné le contraste marqué entre l'agriculture des paroisses du bord du fleuve - accordant une place de choix aux activités laitières - et celle des paroisses plus à l'intérieur des terres ${ }^{22}$. Cette explication ne vaut pas pour les producteurs laitiers des comtés du sud-ouest de la province et de la région immédiate de Québec. Dans la première de ces régions, il existe quelques fabriques importantes, traitant le lait d'une centaine de producteurs. Mais surtout, l'essentiel des installations de concentration et d'expédition de crème de la province - le plus souvent d'anciennes fabriques - s'y trouve localisé ${ }^{23}$. Ces producteurs laitiers disposent manifestement d'autres débouchés pour leur lait, marchés que les corrélations imparfaites entre la cartographie du réseau de fabriques et celle des ventes de lait et de crème aident à préciser.

Les proportions de lait vendu sont importantes là où les installations de transformation - les fromageries notamment - sont nombreuses, mais aussi, là où les villes offrent un bassin substantiel de consommateurs (figure 5). Tel est le cas des comtés proches de Montréal et de Québec. La répartition des fortes ventes de crème obéit à une logique autre que celle de la distribution des beurreries (figure 6). Les producteurs des Cantons de l'Est dominent nettement l'ensemble de la province pour les quantités de crème commercialisées. Déjà dans la seconde moitié du XIX ${ }^{\mathrm{e}}$ siècle, le beurre fabriqué dans les Cantons se distinguait par sa grande qualité, ce qui lui valait un prix supérieur chez les grossistes exportateurs et chez les détaillants de Montréal. Peu avant la Première Guerre mondiale, à la faveur d'un adoucissement des tarifs américains sur les produits laitiers, les cultivateurs des Cantons établissent un commerce lucratif de crème et

21 Mes tentatives de calcul du rapport du nombre de patrons au nombre de fabriques pour chaque comté se sont avérées infructueuses, en raison des nombreuses ambiguittés que présentent les données des annuaires statistiques. Le rapport que j'emploie pour cette carte ne vise qu'à illustrer la couverture (densité) du réseau d'installations plutôt que le degré de participation des exploitants.

22 Bruno Jean, Agriculture et développement dans l'Est du Québec (Sainte-Foy, Presses de l'Université du Québec, 1985).

23 Cinquante des 78 stations d'expédition de lait et de crème actives en 1927 sont situées dans les comtés de Brome, Compton, Huntingdon, Missisquoi, Shefford, Sherbrooke et Stanstead. Annuaire statistique de la province de Québec, 1928, 268. 
Figure 4

Nombre de fermes laitières

par fabrique de beurre et de fromage, 1920

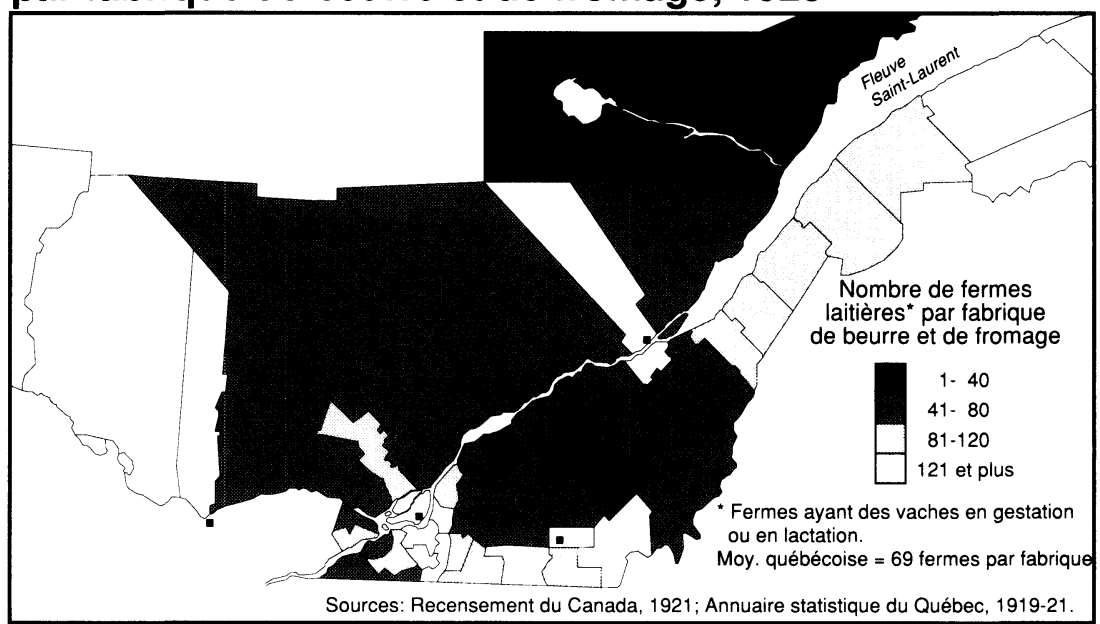

Figure 5

Vente de lait, 1920

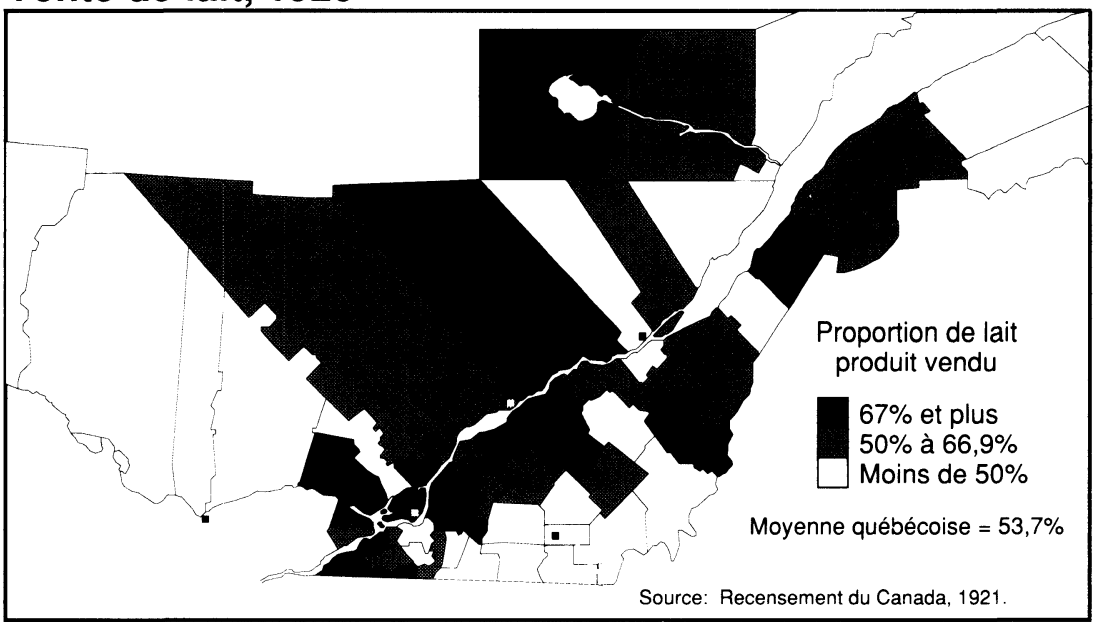


Figure 6

Crème vendue par ferme laitière, 1920

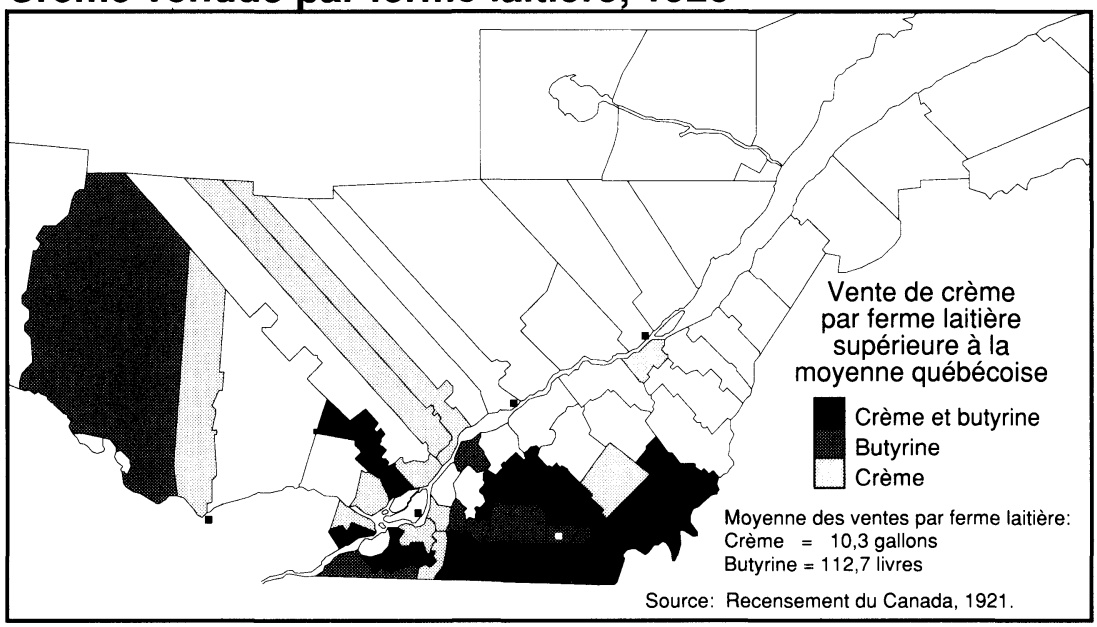

Figure 7

Production et vente de beurre de ménage, 1920

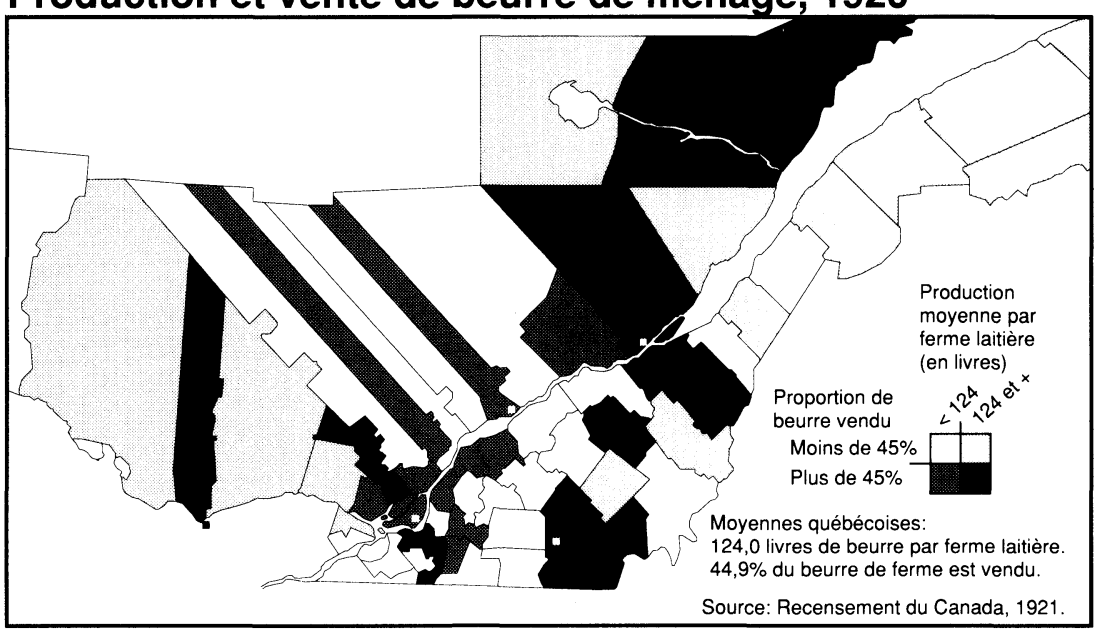


de gras de beurre (butyrine) avec les grands marchés de la NouvelleAngleterre $^{24}$. La vente de la crème suivant sa teneur en matière grasse atteste la forte concentration spatiale de cette activité: à eux seuls, les comtés de Stanstead, de Missisquoi, de Compton, de Shefford et de Brome totalisent plus de $50 \%$ du volume de la butyrine vendue dans la province.

Les producteurs laitiers ne font pas juste l'expédition de lait et de crème sous forme liquide: un important commerce de beurre de ménage subsiste en 1921. En effet, la diffusion d'écrémeuses-séparatrices au tournant du siècle permet le maintien de cette activité sur la ferme ${ }^{25}$. Produit d'abord pour la consommation domestique, ce beurre est aussi acheminé vers les consommateurs des villes et des villages: $45 \%$ de la production québécoise est effectivement commercialisée, vendue aux marchands locaux, aux agents exportateurs ou directement sur les marchés publics. De fait, les quantités vendues sont importantes dans les comtés proches de Montréal, de Québec, d'Ottawa et de Sherbrooke (figure 7).

Au début des années 1920, il existe donc trois grands marchés pour le lait et ses dérivés. Basée sur le commerce d'exportation de fromage et de beurre vers le marché britannique, la vente de lait et de crème aux fabriques domine dans les régions qui ne disposent pas d'un accès privilégié aux grands marchés urbains du Québec et des États-Unis. L'expédition de crème vers les villes de la NouvelleAngleterre demeure l'apanage des Cantons de l'Est et d'un nombre vraisemblablement important de producteurs transitant par Montréal. Le marché des villes de la province, Montréal en tête, constitue le troisième grand débouché pour le lait et la crème.

Dans chacun des cas, les conditions de commercialisation diffèrent considérablement: ce ne sont pas les mêmes intermédiaires, ni les mêmes calendriers de livraison et de paiement, ni les mêmes prix de vente, ni les mêmes exigences quant à la manière de faire et de livrer le lait. Par exemple, le prix obtenu par le producteur pour le lait livré à Montréal excède de plus du tiers celui payé par les fabriques: en 1920 , les compagnies distributrices de la ville offraient 35 cents pour le gallon de lait livré tandis que les fabriques québécoises ne don-

24 La crème extrêmement riche en gras de beurre entre sur le marché américain sans payer les tarifs élevés de la crème et du beurre. La même écrémeuse-séparatrice, qui allège la préparation du beurre sur la ferme, pouvait aussi servir à concentrer la crème pour son expédition aux États-Unis; cette pratique est dénoncée par les promoteurs de l'industrie laitière québécoise, car elle prive les fabriques de matière première.

25 En 1921 comme en 1931, les exploitations familiales fabriquent en moyenne 125 livres de beurre. 
naient en moyenne que 22 cents $^{26}$. Dans son témoignage devant le comité permanent de l'agriculture et de la colonisation en 1933, Peter D. McArthur, président de la Montreal Milk Producers Association, rappelle que les producteurs laitiers vendant à la ville avaient coutume, dans les années 1920, d'établir leur prix de vente en ajoutant un dollar au prix courant du cent livres de lait offert par les fabriques de fromage de la région montréalaise ${ }^{27}$. Par ailleurs, au moment de la négociation du prix de vente, les producteurs laitiers doivent affronter des interlocuteurs passablement différents: dans un cas, ils font affaire avec quelques petits fabricants qui cherchent, bon an, mal an, à conserver leurs fournisseurs; dans l'autre, le producteur fait face à une quarantaine de compagnies distributrices relativement solidaires ${ }^{28}$ qui cherchent à obtenir un approvisionnement régulier et peu coûteux.

Ces trois grands marchés se partagent une même matière première. S'il existait en 1920 un semblant d'équilibre dans la répartition des volumes laitiers entre chaque débouché, des incidents comme l'épidémie de typhoïde touchant Montréal en 1927 soulignent sa grande précarité. Au début du mois de mars de cette année apparaissent les premiers cas de typhoïde dans la ville de Montréal. Vérifications faites par le service de santé de Montréal, le lait provenant de la Montreal Dairy Co. Ltd s'avère le vecteur de la maladie ${ }^{29}$. Aussitôt connue la source de l'épidémie, les autorités sanitaires américaines ferment la frontière au lait provenant d'un rayon de 200 milles autour de Montréal, le tout au grand contentement des producteurs de la Nouvelle-Angleterre ${ }^{30}$. De façon immédiate, l'embargo

26 Bureau fédéral de la Statistique, Commerce intérieur, Prices and Price Indexes, 1920, «Milk, f.o.b. Montreal», Esdras Minville dir., L'agriculture (Montréal, Fides, 1943), 517.

27 Chambre des Communes, Comité permanent de l'agriculture et de la colonisation, Lait et produits laitiers (1933), 28.

28 Même si une vingtaine de laiteries montréalaises forment un front commun lors des négociations, cette coalition reste fragile, en raison de la différence de taille entre ces compagnies. Quelques grandes laiteries, telles que la Guaranteed Pure Milk, J.-J. Joubert, Montreal Dairy, Elmhurst Dairy, Borden, orientent à toutes fins pratiques les positions de l'association. Faute d'espace, je ne peux traiter dans ce texte de l'essor du réseau de distribution de lait à Montréal, ni du phénomène de concentration des laiteries distributrices de Montréal durant cette période.

29 J'ai suivi le déroulement de cette crise dans les journaux montréalais. J'ai également consulté les rapports du Conseil d'hygiène de la province de Québec (1927-1929) ainsi que les numéros du Bulletin d'hygiène, publiés par la ville de Montréal, pour l'année 1927.

30 Le consul américain à Montréal, Albert Halstead, nie toute pression de la part de l'industrie laitière américaine. Il ajoute, dans cette correspondance qu'il fait parvenir au Secrétariat d'État quelques jours après l'imposition de l'embargo (31 mars 1927): «... that everyone whom I have met since the embargo has expressed approval of the action of the United States Government and because of the feeling that it will lead to improving sanitary conditions amongst Montreal dairies, will make more efficient the sanitary administration throughout the province, and in general be a salutary lesson (le souligné est de nous). It should not be thought, 
touche les expéditeurs de crème des Cantons de l'Est et de l'Est ontarien qui, ironiquement, n'ont pas ou peu de contact avec le marché montréalais. C'est sur ce même marché que les producteurs de ces régions vont provisoirement se replier et y provoquer une situation de surplus de lait et de crème.

Loin de se résorber, ce surplus d'approvisionnement de lait ne cessera de prendre de l'ampleur longtemps après la fin de l'épidémie. Citons ici le témoignage de $\mathrm{M}$. William Moore, président et directeur général de la United Dairymen Co-operative Company de Montréal devant le comité permanent de l'agriculture et de la colonisation de la Chambre des Communes en 1933:

Cet accroissement [des arrivages] est attribuable d'abord à la cessation, il y a deux ans, des expéditions considérables de lait et de crème aux États-Unis. Les cultivateurs des comtés du sud de la province de Québec, ayant passé l'inspection très rigide prescrite par Washington pour leur permettre d'expédier du lait aux États-Unis, pouvaient facilement passer l'épreuve nécessaire pour expédier à Montréal. [...] nous avons subi la répercussion des bas prix du beurre et du fromage l'automne dernier. L'hôtel de ville est inondé de demandes de cultivateurs qui cherchent à faire inspecter leurs fermes en vue d'expédier leur lait à Montréal. ${ }^{31}$

Ainsi l'adoption de nouvelles dispositions d'inspection et de contrôle sur l'importation des produits laitiers (à la fin d'avril 1927) et les hausses des tarifs douaniers (dès 1930) restreignent l'accès au marché américain. Par ailleurs, le ralentissement des expéditions de fromage sur le marché anglais affecte tant les fabricants que leurs fournisseurs. Dès lors, un nombre grandissant de producteurs laitiers tenteront de venir vendre leur lait à Montréal, là où la demande est croissante, là où le marché est le plus rémunérateur.

\section{LES FOURNISSEURS DE LAIT DE MONTRÉAL}

Jusqu'aux années 1880 , une large fraction du lait consommé à Montréal est produite sur place, à l'instar des productions maraîchères,

\footnotetext{
however, that there is a large proportion of insanitary dairies in Quebec. Many of the farmers are not up-to-date in their methods and doubtless much of the milk is unclean but there are many sanitary dairies, some in the eastern townships and some around Athelston on the New York Central Line from Montreal to Malone, and clean dairies can result from proper education and attention to the subject.» United States of America, Department of State, Papers relating to the Foreign Relations of the United States, 1928, volume 2, (1943), 110.

31 Chambre des Communes, Comité permanent de l'agriculture et de la colonisation, Lait et produits laitiers (1933), 32.
} 
par les producteurs établis à l'intérieur des limites de la ville et par ceux des paroisses de l'île de Montréal $^{32}$. Outre ces exploitations agricoles, plusieurs familles de la ville vont pratiquer l'élevage de porcs, de volailles et de vaches laitières ${ }^{33}$. Le prix d'achat et les frais d'entretien d'une vache limitent cette avenue aux familles plus «aisées».

L'essor de la population urbaine aura le double effet d'accroître la demande de lait et d'exercer une pression sur le territoire occupé par les étables et les enclos, ce qui a pour résultat dans les deux cas de repousser les activités de production laitière au-delà du périmètre municipal. En 1891, l'inspecteur du lait de la ville note avec une extrême satisfaction la diminution du nombre d'étables sur le territoire municipal: il reste moins de 120 «laitiers» surtout localisés dans les quartiers de Sainte-Marie, Saint-Jean-Baptiste et Saint-Antoine. Et le même inspecteur de poursuivre en constatant «...l'augmentation de ceux qui vont se fixer dans les paroisses éloignées de l'île. Ce mouvement ne saurait être trop loué; car il devra avoir un double avantage pour l'économie et pour la salubrité. ${ }^{34}$ Le mouvement en question n'est pas récent car ces paroisses de l'île de Montréal sont depuis longtemps «branchées» sur le marché urbain. À en croire les statistiques recueillies par le bureau municipal d'inspection du lait pour cette même année, ces localités fournissent déjà plus de la moitié du volume consommé annuellement dans la ville: parmi elles se démarquent les paroisses de Saint-Laurent, du Côteau Saint-Louis, de Longue-Pointe et de Saint-Léonard-de-Port-Maurice.

C'est de moins en moins la proximité du marché urbain que l'accessibilité offerte par le chemin de fer qui déterminera les contours du bassin d'expéditeurs. Des quantités sans cesse plus importantes de lait arrivent dans les gares montréalaises en provenance de fournisseurs éloignés. Cette part croissante du volume quotidien est

32 Pour une évaluation de ces activités dans la première moitié du XIX ${ }^{e}$ siècle, voir JeanClaude Robert, «Activités agricoles et urbanisation dans la paroisse de Montréal, 1820-1840", François Lebrun et Normand Séguin dir., Sociétés villageoises et rapports villes-campagnes au Québec et dans la France de l'Ouest XVIIe-XXe siècles. Actes du colloque franco-québécois de Québec (1985). (Trois-Rivières, Centre de recherche en études québécoises, Université du Québec à Trois-Rivières, 1987), 91-100.

33 Bettina Bradbury décrit ces pratiques dans son article «Pigs, Cows, and Boarders: Non-Wage Forms of Survival Among Montreal Families, 1861-1891», Labour/Le travail, 14 (automne 1984): 9-46.

34 «Rapport sur l'état sanitaire de la Cité de Montréal et sur les opérations de sa commission d'hygiène ainsi que sur la statistique mortuaire pour l'année 1891», Rapports sur les comptes de la Corporation de la Cité de Montréal et rapports des chefs de département pour l'année 1891, 67. 
LA DIFFÉRENCIATION DES PRODUCTEURS LAITIERS...

\section{TABLEAU 1}

Expéditeurs de lait et de crème vers Montréal selon le lieu d'origine et le mode d'expédition

(1913-1914)

\begin{tabular}{|c|c|c|c|c|c|}
\hline \multirow[t]{2}{*}{ Comté } & \multirow[t]{2}{*}{ Lait } & \multirow[t]{2}{*}{ Crème } & \multirow{2}{*}{$\begin{array}{l}\text { Via postes } \\
\text { d'écrémage }\end{array}$} & \multicolumn{2}{|c|}{ Total } \\
\hline & & & & $\mathbf{N}$ & $\%$ \\
\hline Île de Montréal & 406 & - & - & 406 & 10,9 \\
\hline Île-Jésus & 68 & - & 310 & 378 & 10,1 \\
\hline Terbonne & 119 & 18 & 165 & 302 & 8,1 \\
\hline Deux-Montagnes & 127 & 33 & 115 & 275 & 7,4 \\
\hline L'Assomption & 139 & 48 & 17 & 204 & 5,5 \\
\hline Argenteuil & 190 & 5 & - & 195 & 5,2 \\
\hline Vaudreuil & 88 & 1 & - & 89 & 2,4 \\
\hline Soulanges & 5 & 1 & - & 6 & 0,1 \\
\hline Rive nord & 668 & 106 & 297 & 1071 & 28,7 \\
\hline Huntingdon & 132 & 12 & 183 & 327 & 8,8 \\
\hline Châteauguay & 186 & 85 & - & 271 & 7,3 \\
\hline Saint-Jean & 84 & - & 92 & 176 & 4,7 \\
\hline Chambly & 57 & - & - & 57 & 1,5 \\
\hline Beauharnois & 16 & 6 & - & 22 & 0,6 \\
\hline Laprairie & 12 & 6 & - & 18 & 0,5 \\
\hline Napierville & 7 & 1 & - & 8 & 0,2 \\
\hline \multicolumn{6}{|l|}{ Rive sud, } \\
\hline à l'ouest du Richelieu & 494 & 110 & 275 & 879 & 23,6 \\
\hline Missiquoi & 33 & 6 & 158 & 197 & 5,3 \\
\hline Richmond & 67 & 35 & - & 102 & 2,7 \\
\hline Bagot & 7 & 84 & - & 91 & 2,4 \\
\hline Drummond & 14 & 67 & - & 81 & 2,2 \\
\hline Nicolet & - & 39 & 41 & 80 & 2,1 \\
\hline Shefford & 9 & 46 & - & 55 & 1,5 \\
\hline Rouville & 54 & - & - & 54 & 1,4 \\
\hline Saint-Hyacinthe & 1 & 31 & - & 32 & 0,9 \\
\hline Brome & 11 & 4 & - & 15 & 0,4 \\
\hline Sherbrooke & 15 & - & - & 15 & 0,4 \\
\hline \multicolumn{6}{|l|}{ Rive sud, } \\
\hline à l'est du Richelieu & 211 & 312 & 199 & 722 & 19,3 \\
\hline Ontario & 249 & 26 & - & 275 & 7,4 \\
\hline Total & 2096 & 554 & 1081 & 3731 & 100,0 \\
\hline
\end{tabular}

Source: F.C. Harrison, A. Sauvage et W. Sadler, The Milk Supply of Montreal: A Report of a Bacteriological Investigation of the City's Milk Supply 1913-1914, SteAnne-de-Bellevue, Macdonald College (McGill University), 1914, p. 14. 
évaluée en 1891 à environ 20\%. Une vingtaine d'années plus tard, les producteurs laitiers des comtés ceinturant la ville accaparent la quasitotalité du marché d'approvisionnement du lait de consommation. Une enquête sur la qualité du lait distribué à Montréal, menée en 19131914 par des professeurs du collège Macdonald de Sainte-Anne-deBellevue, permet de tracer, de façon assez fidèle, le pourtour du réseau d'approvisionnement en lait et en crème ${ }^{35}$ (tableau 1). Les producteurs des paroisses rurales de l'île de Montréal comptent pour $11 \%$ des expéditeurs; une proportion semblable vaut pour les paroisses de l'île de Laval. Le gros des fournisseurs est au-delà. Deux zones se détachent clairement: celle de Châteauguay-Huntingdon, au sud du fleuve Saint-Laurent, et celle que forment les quatre comtés au nord de l'île Jésus, soit Argenteuil, Deux-Montagnes, Terrebonne et l'Assomption. Notons également la présence d'un nombre appréciable d'expéditeurs de l'Est de l'Ontario $(7,4 \%)$. Compte tenu de ce que nous avons vu plus haut, la représentation relativement faible des Cantons de l'Est et de la région de Saint-Hyacinthe ne surprend guère. Dans le premier cas, les échanges entre les Cantons de l'Est et les États-Unis détournent les expéditeurs du marché montréalais. Quant à la grande région maskoutaine, il semble que les producteurs misent davantage sur la transformation du lait en beurre et en fromage que sur son expédition sous forme liquide, se conformant ainsi aux conseils des directeurs de la Société d'industrie laitière.

Dans un bref article paraissant en 1924, André d'Anjou, analyste au laboratoire municipal de Montréal, confirme et précise cette répartition des expéditeurs: il tient compte cette fois des quantités de lait reçues à la ville:

On ne retrouve plus aujourd'hui sur toute l'étendue de l'île de Montréal qu'environ 500 vaches fournissant à la ville un millier de gallons de lait par jour. Ce millier de gallons représente aujourd'hui un soixantième de la consommation quotidienne. [...] Un tiers du lait consommé quotidiennement nous vient de l'Ontario (Prescott, Brockville et leurs environs). Un autre tiers nous est fourni par les seuls comtés de Châteauguay, Huntingdon, Deux-Montagnes et Argenteuil; l'autre tiers nous est expédié d'un peu partout, savoir: l'île Jésus, L'Assomption, Longueuil, Boucherville, les Cantons de l'Est (Sherbrooke, Richmond et leurs environs), Trois-Rivières, Malone, Vermont, etc. ${ }^{36}$

35 F. C. Harrison et al., The Milk Supply of Montreal: A Report of a Bacteriological Investigation of the City's Milk Supply 1913-1914 (Ste-Anne-de-Bellevue, Macdonald College (McGill University), 1914). Ce rapport reste muet sur les volumes expédiés, ce qui permettrait de nuancer l'importance des comtés fournisseurs.

36 «La question du lait», Bulletin des Agriculteurs, 21 février 1924, 5. 
Dans ce bassin de 150 kilomètres de diamètre, combien de producteurs expédient les 55000 à 60000 gallons de lait que la ville consomme quotidiennement ${ }^{37}$ ? Peu avant la Première Guerre mondiale, les auteurs du rapport du collège Macdonald dénombrent 2096 expéditeurs de lait et 1635 fournisseurs de crème. Dix années plus tard, l'analyste municipal d'Anjou mentionne 4000 fournisseurs de lait. En 1930, le bureau d'inspection du lait de la ville de Montréal agrée les permis de 4358 expéditeurs de lait et près de 1900 expéditeurs de crème ${ }^{38}$.

Cette question des effectifs mène à celle de la composition du groupe des fournisseurs. En guise de conclusion à son compte rendu de la grève de 1929, R.-M. Migner décrit les producteurs de lait de la région de Montréal comme

les agriculteurs les plus solidement intégrés à l'économie de marché de la ville [...] Plus que tous les autres cultivateurs de la province, ils devaient subir la tyrannie de l'offre et de la demande et affronter des intermédiaires capitalistes. Prisonniers d'un système de monoculture, ils étaient les plus susceptibles de souffrir d'une augmentation de leurs coûts de production. ${ }^{39}$

Selon Migner, les producteurs laitiers expédiant à Montréal se distinguent par le degré élevé de spécialisation de leurs activités et par leur forte intégration au marché. J'ai cherché à vérifier ces caractéristiques en tentant d'évaluer l'importance des ventes de lait parmi les autres productions - en distinguant lorsque c'est possible le débouché - et en regardant de plus près les associations formées par ces producteurs pour mieux contrôler la mise en marché.

Pour apprécier la place de la production laitière dans le système d'exploitation, il faut se rendre, en quelque sorte, sur les fermes. Les recensements canadiens ne permettent pas de distinguer précisément l'agencement des cultures et des élevages sur les exploitations agricoles. Par contre, des sources plus spécifiques, telles les enquêtes sur les coûts de production commandées par les ministères de l'Agriculture du Canada et du Québec et les rapports du concours du Mérite agricole de la province de Québec ${ }^{40}$, fournissent de précieuses

37 «Lait et crème consommés dans quelques cités et villes en 1921», Annuaire statistique de la province de Québec, 1922, 206. 1933,30

38 Chambre des Communes, Comité permanent de l'agriculture et de la colonisation,

39 Robert M. Migner, Le monde agricole québécois et les premières années..., 286.

40 Danielle Noiseux utilise ces rapports afin d'obtenir des indications sur la modernisation des exploitations agricoles des comtés de Laprairie et Napierville. La modernisation agricole dans les comtés de Laprairie et Napierville, 1920-1970: les choix des 
indications sur l'organisation et le degré de commercialisation des productions. Les résultats de chaque concours du Mérite agricole font l'objet d'un rapport publié dans lequel on trouve les commentaires généraux du jury et les descriptions de la plupart des exploitations visitées. Le traitement de ces descriptions ne va pas sans problème. D'une part, l'inégale distribution des candidats suivant les comtés affecte la représentativité du groupe ${ }^{41}$. D'autre part, les descriptions sont loin d'être également minutieuses et détaillées pour tous les candidats. Dans l'ensemble cependant, le secrétaire brosse un court portrait des points saillants de l'exploitation, accompagné de plans et de photographies des bâtiments, des champs, du jardin ou des animaux primés. Bien que nous n'ayons pas affaire à un groupe représentatif, ni spatialement, ni en termes de composition, de producteurs agricoles de la région de Montréal, ces portraits d'exploitations permettent de préciser la place des activités laitières dans le système de production et de formuler un certain nombre d'observations concluantes sur la différenciation des producteurs laitiers suivant le débouché.

À l'exception d'une poignée de producteurs maraîchers spécialisés de l'île de Montréal et des comtés de Napierville et de Laprairie, il ne se trouve pas d'exploitations des concurrents du Mérite agricole de 1920 , de 1925 et de 1930 sans bétail laitier. La production laitière est associée le plus souvent à l'élevage (bovin et porcin), à la culture du tabac, du foin, ou des fruits et légumes ${ }^{42}$. En 1920, la production et la vente du lait sont mentionnées explicitement dans moins de la moitié des descriptions (23 concurrents); quatorze producteurs font la vente de lait et de la crème en nature à la ville tandis que les neuf autres alimentent principalement les beurreries et les fromageries.

Les descriptions plus étoffées des concours de 1925 et de 1930 permettent de dresser une répartition des producteurs laitiers suivant le

producteurs, mémoire de maîtrise, Université du Québec à Montréal, août 1985. Pour l'historique du concours, voir J.-B. Roy, Histoire du Mérite agricole (Agriculture Québec, 1978) et Firmin Létourneau, «Histoire de l'agriculture», Canada français (1968), chapitre 9, 225-237. 41 Sur les 295 concurrents inscrits aux concours tenus dans la région de Montréal pendant les années 1920 (concours de 1920, de 1925 et de 1930), près du tiers (90 candidats) proviennent du comté de Deux-Montagnes: voyons là le résultat des encouragements formulés par les professeurs de l'Institut agricole d'Oka. Suivent les comtés de Soulanges (31), Vaudreuil (27), Laprairie (26) et l'Assomption (25). Par ailleurs, la faible participation des cultivateurs des comtés du sud-ouest de Montréal, en particulier ceux de Châteauguay et de Huntingdon, nous prive d'une représentation pour cette région où résident de nombreux expéditeurs de lait.

42 Soit les grands axes de production commerciale qu'avait observés Raoul Blanchard pour la région: Raoul Blanchard, L'Ouest du Canada français. Montréal et sa région (Montréal, Beauchemin, 1953), 89-125. Voir également la description des zones agricoles de la province dans le programme du ministre J.-L. Perron, annexée au rapport du ministère de l'Agriculture de 1929-1930. 
TABLEAU 2

Recettes et part de la vente de lait de 57 exploitations de la région de Montréal (1925)

\begin{tabular}{|c|c|c|c|c|c|}
\hline \multirow{2}{*}{$\begin{array}{l}\text { Recettes de la } \\
\text { vente de lait }\end{array}$} & \multicolumn{5}{|c|}{ Part de la vente de lait dans les recettes totales } \\
\hline & $\begin{array}{c}\text { Moins de } \\
33 \% \\
\end{array}$ & $\begin{array}{l}33 \text { à } \\
49 \% \\
\end{array}$ & $\begin{array}{l}50 \text { à } \\
66 \%\end{array}$ & $\begin{array}{c}67 \% \text { et } \\
\text { plus }\end{array}$ & Total \\
\hline Mons de $1000 \$$ & 13 & 12 & 1 & - & 26 \\
\hline $1000 \$$ à $1999 \$$ & 3 & 2 & 5 & 3 & 13 \\
\hline $2000 \$$ à $2999 \$$ & - & 1 & 4 & 2 & 7 \\
\hline $3000 \$$ et plus & - & & 7 & 4 & 11 \\
\hline Total & 16 & 15 & 17 & 9 & 57 \\
\hline
\end{tabular}

Source: Rapport du concours du Mérite agricole de la Province de Québec, 1925.

débouché. En 1925, environ trois concurrents sur dix vendent leur lait à la ville; cinq années plus tard, la moitié des participants font pareil commerce. Le rapport de la compétition de 1925 donne le sommaire des recettes et des dépenses de 57 exploitations, sommaire suffisamment précis pour permettre une évaluation grossière de la part occupée par les produits de la laiterie dans les revenus de la ferme (tableau 2). Il n'est pas surprenant de voir la proportion de recettes tirées de la vente du lait croître avec la valeur de l'ensemble des recettes. Si l'on tient compte du débouché, une tout autre image apparaît. Les onze producteurs qui vendent plus de $3000 \$$ de lait trouvent leur marché à Montréal ${ }^{43}$. En d'autres termes, pour 20 des 24 exploitations effectuant la vente de lait aux compagnies distributrices de Montréal, les recettes de la laiterie représentent plus de la moitié des recettes totales de l'exploitation. J'ai fait le même calcul pour les producteurs qui vendent aux beurreries et aux fromageries et pour ceux qui expédient seulement de la crème ( 33 exploitants): les ventes de lait et de crème ne dépassent jamais $1500 \$$ et composent entre le tiers et la moitié des recettes totales de l'exploitation.

Ces données suggèrent l'existence de différences significatives entre les producteurs sur le plan des recettes tirées de la vente de lait: de grosses recettes et une spécialisation laitière relativement poussée semblent caractériser les fournisseurs laitiers de Montréal. D'autres indices de cette différenciation peuvent être présentés. Les conditions avantageuses de ce marché dans les années 1920 poussaient ces

43 J'ai retenu les cas mentionnant explicitement la vente de lait à la ville ou à une compagnie distributrice de Montréal. 
producteurs à concentrer leurs efforts sur l'augmentation rapide du volume de la production. Plutôt que de chercher à accroître la productivité du cheptel par le contrôle laitier et par l'amélioration des soins et de l'alimentation, certains expéditeurs de lait cessent de pratiquer l'élevage, comme ils l'avaient déjà fait pendant la Première Guerre mondiale, pour renouveler leur troupeau par l'achat et la vente de vaches laitières. Tel Ulric Gohier qui

achète des vaches laitières, pour la plupart, aux abattoirs, les garde pour une ou deux périodes de lactation, rarement davantage et les revend ensuite pour la boucherie. Ce système a pu avoir du bon, surtout chez les connaisseurs de bétail laitier; avec les nouveaux réglements du conseil de ville de Montréal, qui obligent tous les fournisseurs de lait à avoir des troupeaux tuberculinisés, il sera difficile à pratiquer. ${ }^{44}$

Cette pratique existe encore en 1929-1930, quoiqu'en régression:

Il y a quelques années, il ne se faisait pas d'élevage sur cette ferme. On achetait des vaches «toutes faites» et on les revendait après une période ou deux de production. Cette pratique, avec les règlements plus sévères du service de l'Hygiène montréalais, avec les aléas du marché des vaches laitières, présentait des inconvénients qui ont fait réfléchir sérieusement MM. Husereau, père et fils. On se décida en 1925 de faire de l'élevage et d'avoir un troupeau tuberculinisé. ${ }^{45}$

Je reviendrai plus loin sur la question des effets des règlements municipaux. Pour l'instant, signalons un certain nombre d'activités connexes au commerce du lait à la ville. Certains producteurs assument le rôle de coordonnateur du réseau d'expédition ou agissent à titre d'agent pour les distributeurs montréalais. George S. Armstrong de Lachute est un de ces producteurs qui, en plus d'expédier son lait à la compagnie J.-J. Joubert de Montréal, «...achète aussi une quantité assez considérable de lait pour la même compagnie». On apprend dans le rapport de 1929-1930 qu'il «...fait aussi le transport du lait pour un certain nombre de fournisseurs de leurs fermes à la station de chemin de fer $»^{46}$. Signalons finalement le cas d'une poignée de producteurs qui effectuent la vente au détail dans les villes et les villages comme

44 Description de l'exploitation de Ulric Gohier, Concours du Mérite agricole... (1925), 25-26. Voir aussi celles de William-J. Hodge, Osias Husereau, Thomas Deslauriers, Ulric Debien, René Lesage, tous des producteurs de lait vendu nature à la ville de Montréal.

45 Hervé Husereau, Concours du Mérite agricole, 1929-1930, 60.

46 George S. Armstrong, Concours du Mérite agricole, 1925 et 1929-1930. 
Valleyfield, Charlemagne, Saint-Jérôme, ou de façon saisonnière dans les endroits de villégiature ${ }^{47}$.

À travers les descriptions des rapports du Mérite agricole, il appert que les producteurs qui expédient du lait à Montréal tirent un revenu substantiel - sinon l'essentiel de leur revenu - de ce commerce. Cette spécialisation relative des activités exige de leur part des efforts constants pour pallier l'augmentation des coûts de production et les fluctuations des prix agricoles. Plusieurs stratégies sont utilisées pour y parvenir. Par exemple, les cultivateurs peuvent hausser le niveau de productivité de leur troupeau afin de profiter des prix élevés de la saison d'hiver. De même, la formation d'associations de producteurs spécialisés leur permet d'influencer les conditions de production et de mise en marché. C'est par la voie de ces regroupements qu'ils réussissent à prendre part à la fixation du prix de gros du lait. Par ailleurs, les associations leur servent également d'outil pour se protéger contre l'intense compétition qui existe entre les fournisseurs qui approvisionnent la ville. Ce dernier aspect est bien illustré par l'histoire des deux associations impliquées dans les événements d'octobre 1929.

Robert Migner dépeint la Montreal Milk Producers Association (MMPA) comme fermée, jalouse de ses prérogatives, largement anglophone parce que comptant parmi ses membres une majorité d'agriculteurs ontariens ${ }^{48}$. En fait, ce regroupement d'expéditeurs de lait est fondé par des producteurs laitiers des comtés de Huntingdon et de Châteauguay au tournant du siècle ${ }^{49}$. Pour des motifs de restructuration et en raison d'un manque chronique de fonds qui affecte le paiement régulier de ses membres, cette première association se réorganise en 1919 sous la loi des sociétés coopératives agricoles ${ }^{50}$. À

47 Ce qui va même jusqu'à comprendre la pasteurisation et l'embouteillage du lait dans le cas de Malcolm Hodge du comté de Jacques-Cartier. Concours du Mérite agricole, 1925.

48 Robert M. Migner, Le monde agricole québécois..., 276-277.

49 Au cours du mois d'octobre 1899, quelque 90 expéditeurs de lait des comtés de Huntingdon, de Châteauguay, de Beauharnois, de Vaudreuil et d'Argenteuil se réunissent à Montréal pour discuter des problèmes de fixation des prix et des coûts de transport. The Huntingdon Gleaner, 12 octobre 1899. Voir dans le même journal les comptes rendus des réunions subséquentes, 15 et 29 mars 1900, et 5 juillet 1900. Une liste des directeurs de la Montreal Milk Shipper's Association paraît pour la première fois dans l'annuaire Lovell de Montréal de 1904-1905. Les renseignements sont plus sommaires par la suite, limités aux seules mentions du nom et de l'adresse des président, secrétaire et trésorier, ce jusqu'en 1918. Des 35 officiers connus de l'association, 28 proviennent des comtés de Beauharnois, Huntingdon, Châteauguay et d'Argenteuil et le reste composé surtout de résidants de l'Est de l'Ontario.

50 Gazette officielle, 1919, 2 414, sous le nom «The Montreal Milk Producers Cooperative Agricultural Association». J'ai retracé les principales pièces de son dossier de fondation, notamment une liste des membres fondateurs, dans les archives du registraire de la province de Québec, relevant du ministère de l'Agriculture (ANQ, Québec, E9-82-10-000/6). Existant jusqu’à la fin des années 1970, la vie de cette coopérative pourrait également être suivie 
cette occasion, les objectifs de l'association, calqués sur ceux de semblables organisations canadiennes et américaines, sont précisés:

A. Favoriser la formation d'un comité composé de producteurs, de consommateurs, d'autorités municipales, fédérales et d'acheteurs. Ce comité doit établir des prix raisonnables et équitables.

B. Surveiller l'approvisionnement et travailler à l'amélioration de la qualité du lait vendu dans les centres.

C. Faire la classification du lait et de la crème et payer ces produits d'après leur valeur propre.

D. Encourager la production de lait de qualité supérieure.

E. S'enquérir du coût de production du lait.

F. Faire connaître les conditions des divers marchés aux producteurs, aux consommateurs et aux acheteurs.

G. Faire le recrutement de membres seulement parmi les producteurs de lait pour la vente en nature.

H. Servir d'agent pour la vente du lait et de la crème, produits par les membres.

I. Faire respecter les prix du lait fixés pour une saison donnée. ${ }^{51}$

L'article $\mathrm{G}$ montre bien la sélectivité de l'organisation: il équivaut à exclure les producteurs laitiers vendant aux fabriques de beurre et de fromage. Malgré cela, des indications éparses qu'on trouve dans les rapports du ministère de l'Agriculture, dans les annuaires statistiques du Québec, dans les rapports du ministère fédéral du Travail sur les organisations coopératives, montrent la progression régulière de ses effectifs: elle compte, en 1919, 524 actionnaires, en 1921, 956 et, en 1929, 1700 répartis dans une vingtaine de sections locales $^{52}$.

\footnotetext{
à travers les procès-verbaux et les autres documents qu'elle a produits, documents repérés par le Montreal Business History Project (Robert Sweeney, Richard Rice et Brian Young, Guide pour l'étude d'entreprises montréalaises et de leurs archives avant 1947 (MBHP, 1978), 174 176). Sur la mutation de cette association, voir «La Société coopérative agricole des producteurs de lait de Montréal», Le Bulletin des agriculteurs, 31 janvier 1920; "Coopérative des producteurs de lait de Montréal», Le Bulletin des agriculteurs, 17 juillet 1920. Voir également la correspondance échangée au mois d'août et de septembre 1919, ANQ-Québec, Ministère de l'Agriculture, E9-82-10-000/6.

51 Bulletin des agriculteurs, 31 janvier 1920.

52 Au milieu des années 1930, le nombre d'actionnaires de cette association s'est accru à plus de 3500 . Voir Canada, Ministère du Travail, Rapport sur l'organisation de l'industrie, du commerce et des professions libérales au Canada, 1922-1935. Incidemment, l'exécutif de l'association consent pendant cette période de nombreux efforts dans le but d'accroître son membership dans les districts francophones du Québec: les comtés de Deux-Montagnes, de Terrebonne, d'Argenteuil et de l'Assomption disposent tous d'au moins une succursale de l'association.
} 
Ce n'est qu'au cours des années 1920 que la MMPA s'impose comme principal interlocuteur auprès des distributeurs de lait et des autorités de la ville. L'entente verbale conclue bi-annuellement sur les conditions de livraison et de paiement du lait, qui tient lieu de contrat entre les fournisseurs et les laiteries, est généralement respectée par les parties. C'est qu'elle réussit très rapidement à regrouper les plus gros expéditeurs de lait de la région ouest de Montréal: dans un mémoire présenté au gouvernement durant la grève de 1929 , le représentant des compagnies distributrices de la ville évalue à $60 \%$ la part de l'approvisionnement que fournissent les 1700 membres de la MMPA ${ }^{53}$. De surcroît, elle adopte une position de négociation particulièrement conciliatrice. À preuve, l'association des laiteries de Montréal et la MMPA vont partager les services du même secrétaire-coordonnateur ${ }^{54}$.

En octobre 1929, l'Association des producteurs laitiers de la province de Québec (APLPQ) vient brouiller l'harmonie de ces négociations. Cette nouvelle organisation voit le jour lors d'une assemblee tenue le 17 septembre 1928 dans une école de Ville-Saint-Laurent ${ }^{55}$. Sa création procède de la volonté de regrouper les producteurs laitiers des environs de Montréal, qu'ils soient membres ou non d'organisations locales ${ }^{56}$. L'objectif central de l'APLPQ renseigne de façon indirecte sur la situation de ses membres. Il est de «...réglementer le prix du lait d'après le coût de production [...] de sauvegarder les intérêts de ses membres en obtenant pour le lait un prix qui permette de réaliser au moins un bénéfice raisonnable» ${ }^{57}$. Un an plus tard, presque jour pour jour, au moment des négociations saisonnières qui mèneront à la grève, cet objectif sera repris et respecté à la lettre. Pour

53 Québec, Documents parlementaires, 1931, document 25, «Réponse à un ordre de l'Assemblée Législative en date du 12 mars 1930: copie de la correspondance entre le ministre de l'Agriculture et toute personne, depuis 1928, relativement au coût de production du lait....», «Memorandum of Milk Dealers», 4.

54 Chambre des Communes, Comité permanent de l'agriculture et de la colonisation, 1933, 29.

55 La Terre de chez nous, 27 septembre 1928.

56 Les fondateurs proviennent du nord de l'île de Montréal (paroisses de Saint-Laurent et de Saint-Léonard-de-Port-Maurice), de l'île Jésus (paroisses de Sainte-Rose, de SaintFrançois), des comtés de Terrebonne, de l'Assomption et de Deux-Montagnes, et sur la rive sud, des régions de Chambly, de Richelieu, de Verchères et de Rouville, comtés qui ne comptent que quelques succursales de la MMPA. Compte tenu de sa brève existence -- elle disparaît tôt dans les années 1930, - peu d'informations sont disponibles sur ses effectifs, sa structure et sur ses activités. Il est par conséquent impossible de déterminer si le nombre de 3000 membres -mentionné le plus fréquemment dans les sources consultées -- résulte d'une évaluation des effectifs des groupements locaux ou d'un décompte des adhérents ayant payé une cotisation?

57 Ibid. 
justifier le prix pour la saison d'hiver 1929-1930, un administrateur explique que:

Les cultivateurs ont décidé d'élever le prix parce que les engrais chimiques sont haussés de $\$ 7.00$ à $\$ 8.00$ la tonne et que la rareté et la cherté des vaches laitières augmentent de plus en plus: parce que les profits des compagnies sont apparemment trop élevés et que les cultivateurs pourraient avoir une part de bénéfices plus grande: parce que la pasteurisation la tuberculinisation l'installation de glacières, comme le veulent les réglements du bureau d'hygiène de Montréal augmentent les dépenses des producteurs de lait. ${ }^{58}$

Cette revendication pressante liant le prix de vente aux coûts de production donne à penser que l'APLPQ rassemble surtout les nouveaux fournisseurs de lait à la ville, ceux qui délaissent les beurreries et les fromageries de la région. Dans le contexte précis de la réorganisation des marchés du lait des années 1920, tous reconnaissent, comme ce directeur de l'APLPQ, qu'il «n'y a pas de meilleur marché que Montréal pour la vente du lait. On nous demande un lait de bonne qualité. Les autorités nous ont imposé des exigences qui ont fait monter de beaucoup les prix de revient» ${ }^{59}$. En somme, à leur entrée sur le marché montréalais, ces nouveaux fournisseurs doivent se tailler une place aux côtés d'un groupe de producteurs déjà bien installés. Surtout, ils doivent effectuer des ajustements coûteux afin de rendre leurs exploitations conformes aux directives émises par les autorités municipales.

\section{EFFETS DE LA RÉGLEMENTATION DU COMMERCE DU LAIT DE CONSOMMATION}

Les producteurs laitiers et les laiteries distributrices sont loin d'être les seuls à déterminer les conditions de mise en marché du lait dans la ville de Montréal: ils doivent se conformer aux lois et aux règlements mis en place par les divers paliers de gouvernement, en particulier par les autorités municipales (sous la pression des réformistes et des hygiénistes ${ }^{60}$ ). Je traiterai brièvement ici de deux aspects de cette question pour lesquels je dispose d'une documentation satisfaisante: il s'agit du pouvoir municipal d'inspection des établisse-

58 Le Canada, 27 septembre 1929.

59 Le Canada, 19 septembre 1929.

60 Sur ce dernier sujet, voir Thomas R. Pegram, «Public Health and Progressive Dairying in Illinois», Agricultural History, 65,1 (hiver 1991): 36-50. 
ments laitiers et surtout de l'instauration de l'examen obligatoire des troupeaux fournissant le lait à la ville ${ }^{61}$.

C'est en 1876 que les autorités sanitaires de la ville de Montréal se voient confier le mandat d'inspection du lait et des fermes présentes sur son territoire ${ }^{62}$. Afin de contrôler la salubrité et l'adéquation des conditions de production et de manutention, elles peuvent interdire et saisir le lait d'un producteur ou d'un laitier contrevenant aux directives du Conseil provincial d'Hygiène ou aux lois fédérales sur l'adultération des substances alimentaires et sur l'industrie laitière. Le nombre d'inspecteurs et leur territoire d'investigation vont progressivement s'accroître. En 1910, les visites des douze inspecteurs, d'abord limitées aux établissements de la ville, sont étendues à toutes les exploitations qui y expédient du lait. Le contrôle s'effectue à l'aide d'un système de fiches de laitiers remplies en trois exemplaires par les inspecteurs et remises au producteur, au laitier et au bureau d'inspection ${ }^{63}$. Les inspecteurs notent chaque établissement et émettent, le cas échéant, un avis signalant les irrégularités. Ultime recours, ils peuvent interdire aux laiteries de la ville de traiter et de distribuer le lait d'un expéditeur fautif. Les ajustements demandés aux exploitants agricoles portent essentiellement sur la conformité des installations, sur la propreté générale des étables et des laiteries, sur la qualité de l'eau et de la nourriture, sur les modes de traite et de conservation du lait. Le caractère lucratif du débouché montréalais incite le cultivateur à apporter les correctifs nécessaires, non sans pester contre ces inspections pointilleuses ${ }^{64}$.

Après un débat long et animé, un nouveau règlement municipal, voté en 1925 et entrant en vigueur en mars 1926, ajoute deux éléments importants au contrôle du commerce du lait, soit l'interdiction du lait

61 Pour une étude des efforts de réglementation de la qualité du beurre et du fromage pour exportation par la Société d'industrie laitière de la province de Québec, voir Ruth Dupré, «Regulating the Quebec Dairy Industry, 1905-1921: Peeling off the Joseph Label», Journal of Economic History, 50,2 (juin 1990).

62 Cité de Montréal, «Règlement concernant la santé», no 105, 21 décembre 1876.

63 Bulletin mensuel du bureau d'hygiène et de statistique, Cité de Montréal, 1,5 (mars 1915): 2. Le rapport du Collège Macdonald de 1913-1914 reproduit un exemplaire de la fiche utilisée à ce moment. Celle en usage à partir du milieu des années 1920 est annexée au rapport du Comité permanent de l'agriculture et de la colonisation, Lait et produits laitiers (1933), 792793.

64 Citons encore une fois J.-N. Ponton: «...l'inspecteur de la ville s'amène. Il lui [le producteur] fait remarquer qu'il est entré des mouches dans son étable, que le plancher n'est pas convenablement ciré, que les rideaux de cretonne des fenêtres ne sont pas de la couleur recommandée par Deschambeault, que les lunettes que portent ses vaches sont d'un vert trop tendre, etc. etc.», Le Bulletin des Agriculteurs, 10 octobre 1929. Il est bon de souligner ici qu'à la même époque, les règlements américains sont plus sévères que ceux de Montréal. 
provenant d'un troupeau n'ayant pas subi l'épreuve de la tuberculine dans les 12 mois et l'obligation de pasteuriser tout le lait mis en vente dans la ville ${ }^{65}$. Cette dernière mesure accélère la transformation et la concentration des compagnies distributrices de la ville ${ }^{66}$. Par contre, l'imposition d'un examen annuel des troupeaux a un effet immédiat sur l'organisation de la production laitière sur la ferme.

Le gouvernement fédéral instaure en 1914 une première série de mesures visant à l'éradication de la tuberculose bovine. Il offre une assistance aux municipalités qui exigent l'épreuve à la tuberculine aux fournisseurs de lait de consommation ${ }^{67}$. Devant le peu de succès de ce premier effort, reposant sur l'initiative des municipalités, le ministère fédéral de l'Agriculture met sur pied en 1919 le système des troupeaux accrédités ${ }^{68}$, s'inspirant directement de l'expérience américaine. Les opérations de détection sont supervisées par les médecins vétérinaires du ministère fédéral de l'Agriculture, lesquels effectuent le test de la tuberculine sur tous les sujets d'un cheptel. Les pertes occasionnées par l'abattage des bêtes réagissant au test sont compensées par le versement au producteur d'une portion de la valeur des animaux. Le certificat d'accréditation est obtenu après la réussite de deux examens consécutifs et peut être ensuite renouvelé annuellement. Pour accélérer et mieux encadrer l'homologation des troupeaux, le ministère de l'Agriculture va délimiter à partir de 1922 des zones réservées, soit des groupes de comté dans lesquels tous les troupeaux sont systématiquement examinés. La première zone réservée de la province de Québec (1924) inclut les trois comtés de Châteauguay, de Huntingdon et de Beauharnois. La zone est élargie à plusieurs reprises pour couvrir en 1927 la partie sud-ouest de la province, y compris les îles Jésus et de Montréal ${ }^{69}$.

Zones réservées ou pas, c'est lorsque la ville de Montréal impose la tuberculinisation en 1925 que les producteurs laitiers s'empressent

65 Certains laits dit spéciaux échappent à cette obligation. Le règlement est adopté le 20 juillet 1925.

66 L'épidémie de typhoïde de 1927 provoque un accroissement de la surveillance municipale de ces installations (une quarantaine). Sept nouveaux inspecteurs sont affectés à cette seule tâche. Bulletin du bureau municipal d' hygiène, 13,8 (novembre-décembre 1927): 5.

67 Chambre des Communes, Comité permanent de l'agriculture et de la colonisation, Addresses delivered before the Committee, 5 mars 1925, «Dr. Hilton's Address», 1-14; Chambre des Communes, Statuts du Canada, Arrêté en conseil, 18 mai 1914 et 16 avril 1917.

68 Chambre des Communes, Statuts du Canada, Arrêté en conseil, 20 septembre 1919.

69 Rapport du ministère de l'Agriculture de la province de Québec, 1927-1928, 123. Sont inclus les comtés de Beauharnois, Châteauguay, Huntingdon, Vaudreuil, Soulanges, Chambly, Verchères, Laprairie, Napierville, Saint-Jean, Iberville, Richelieu, Saint-Hyacinthe, Rouville, Bagot, Missisquoi, Brome, Shefford, les îles de Montréal et Jésus et parties des comtés de Sherbrooke, Richmond, Drummond et Yamaska. 
de demander l'accréditation de leurs troupeaux. Ceci n'ira pas sans problème, ni sans excès. Le programme de compensation ne couvre qu'une partie des frais occasionnés par la destruction des bêtes atteintes et la désinfection de l'étable. Profitant du surcroît de travail des vétérinaires chargés de mener les examens et la lourdeur du mécanisme de vérification, certains producteurs, certains marchands de bétail de la ville et certains propriétaires d'abattoirs vont se livrer à des transactions visant à «blanchir» les animaux réactifs.

La correspondance échangée en 1927 entre le vétérinaire J.-S. Jasmin et les responsables du service de l'élevage du ministère provincial de l'Agriculture, publiée dans les Documents de la Session de 1930, permet de décrire la situation confuse qu'entraîne l'application des nouveaux règlements de la ville ${ }^{70}$. En vertu d'une entente fédéraleprovinciale, une vingtaine de vétérinaires s'affairent quelques jours par mois à effectuer gratuitement l'épreuve de tuberculine chez les producteurs qui font la demande auprès du service provincial de l'élevage. Une zone précise est assignée à chaque vétérinaire: ainsi le secteur couvert par Jasmin est composé de la partie est de l'île de Montréal (Hochelaga et Maisonneuve) et de l'île Jésus, à l'exception de la paroisse de Saint-François-de-Sales.

Dès la mise en marche du programme, des directives peu claires quant à l'homologation des résultats du test provoquent une certaine confusion. C'est que le même médecin vétérinaire travaillant pour le gouvernement peut effectuer l'épreuve aux frais du producteur qui le lui demande, sans égard au comté de résidence de ce producteur. Or, aux yeux des autorités de la ville de Montréal et des ministères de l'Agriculture, seul un test supervisé par le programme fédéral valide le troupeau. Cette fine distinction n'est pas toujours comprise par les producteurs et les médecins vétérinaires chargés d'effectuer les tests. Le docteur Jasmin ne cesse de se plaindre, à la direction du service de l'élevage du ministère de l'Agriculture de Québec, des agissements d'un collègue de Sainte-Thérèse qui effectue à titre privé l'épreuve dans certaines paroisses de l'île de Laval en utilisant les documents, les marques (au fer rouge) et les matricules (tags) du gouvernement.

De même, les fraudes commises pour éviter l'abattage des vaches réactives sont nombreuses et ingénieuses. Certains cherchent à fausser

70 Documents de la Session de Québec, vol. 63, 1930, document no 24, «Copie de la correspondance, lettre, télégramme etc. depuis 1927 entre le gouvernement ou aucun de ses membres et les médecins vétérinaires à son emploi entr'autres le docteur J. S. Jasmin de la cité de Montréal relativement à la tuberculose chez les animaux et à l'épreuve de tuberculine». 
le test en appliquant, par exemple, de l'iode sur la partie témoin ${ }^{71}$ ou du cirage à chaussure sur la marque «UTB». Ces procédés ont le défaut d'être facilement repérables. Lorsque le test a lieu sur le site d'un marché ou dans les parcs des abattoirs, le producteur peut chercher à vendre l'animal testé avant que le résultat ne soit connu. Par exemple, «Ce M. J. Galarneau a refusé de faire brûler ses 3 réacteurs. Maintenant qu'ils les a vendus aux Rév. Sœurs du couvent pensionnaire de Saint-Vincent-de-Paul, il est prêt à faire tuberculiniser de nouveau. ${ }^{72}$

Le trafic des animaux réactifs par les marchands de bétail semble être aussi très important, si l'on en juge par les commentaires du vétérinaire Jasmin. Au lieu d'acheminer les animaux tuberculeux vers les abattoirs, des commerçants les revendent, en maquillant au besoin les résultats du test. La pratique de certains commerçants ontariens, qui se débarrassent des vaches atteintes sur le marché montréalais, est suffisamment répandue pour que le chef du service provincial de médecine vétérinaire suggère l'établissement d'une barrière sanitaire entre les deux provinces ${ }^{73}$. L'enregistrement des animaux, à l'aide de formulaires et de matricules agrafées aux oreilles, n'arrête pas les fraudeurs. Ainsi, Jasmin soupçonne un marchand de bétail de la ville de substituer les étiquettes des vaches ${ }^{74}$. Plus subtile est la méthode que J.-N. Ponton décrit dans le Bulletin des agriculteurs ${ }^{75}$. En injectant de la tuberculine à une vache, on la vaccine en quelque sorte pour une période de soixante jours. Ainsi au moment de l'achat, une vache «vaccinée» par le marchand peut présenter un test acceptable pour ensuite échouer, deux mois plus tard, au même examen chez le cultivateur.

La majorité des producteurs cherchent à se conformer aux règlements de la ville, acceptant les insuffisantes compensations fédérales en échange de l'obligation douloureuse de devoir faire abattre une partie ou l'ensemble de leur cheptel laitier. Déjà, au moment de la visite estivale des juges du Mérite agricole de 1925, plusieurs concurrents rebâtissent leurs troupeaux décimés par les résultats de l'examen ${ }^{76}$; de même, comme nous l'avons noté plus haut, beaucoup

71 J.-S. Jasmin au service de l'élevage du ministère de l'Agriculture, 25 février 1927, 4 mars 1927 et 19 juillet 1927.

72 Ibid., lettre du 28 février 1927. Voir également la lettre du 25 février 1927.

73 Rapport du ministère de l'Agriculture de la province de Québec, 1926-1927, 133.

74 Ibid., lettres du 23 juillet et du 7 août 1927.

75 «Tuberculinisation. Est-on logique?», Bulletin des agriculteurs, 10 octobre 1929.

76 M. William Hodge (Saint-Laurent, comté Jacques-Cartier) «... a d'abord fait subir l'épreuve de la tuberculine à ses bêtes, dont plusieurs ont été trouvées tuberculeuses, puis il s'est décidé à faire l'élevage de ses vaches laitières.» Rapport du concours du Mérite agricole de 1925, 30. Voir aussi les descriptions des fermes de Osias Husereau (Oka, Deux-Montagnes), Théophile Reid (Ormstown, Châteauguay) et Jean-Marie Labelle (Sainte-Rose). 
recommencent l'élevage laitier. Chez certains producteurs, l'abattage du troupeau provoque des situations particulièrement difficiles:

M. George Labelle, Pont-Viau, qui a refusé de laisser brûler ses huit réacteurs, sur onze bovidés, ne l'a point fait avec mauvaise intention: il a vendu ses huit réacteurs et un sain pour la boucherie à ma connaissance, il ne lui reste que deux sains, c'est un pauvre homme, honnête, il est à loyer et a une grosse famille, il s'est trouvé un peu découragé, je vous ferai remarquer que nos commerçants ne paient pas nos réacteurs leurs pleine valeur ils abusent un peu de la bonne foi de nos cultivateurs.

Auriez-vous objection que je marque les 2 animaux sains de ce monsieur afin qu'il puisse continuer de vendre du lait à Montréal: il a très bien désinfecté son étable.

Vos réglements sont là, il est vrai mais c'est plutôt un acte de charité et mettons-nous dans sa position. ${ }^{77}$

Dans ce cas précis, pour contourner la règle, Jasmin intercède auprès de son supérieur. Ce dernier d'ordinaire lui fournit une liste de noms de producteurs à traiter en priorité, par exemple, lorsqu'il lui demande de faire diligence dans l'examen des troupeaux de la municipalité de Saint-Léonard-de-Port-Maurice. Jasmin répond au chef du service d'élevage:

Je me suis mis à la disposition de M. Wilfrid Bastien, Maire de Saint-Léonard tel que le télégramme reçu. Le travail terminé, j'ai continué dans la même paroisse vu que les demandes sont nombreuses et que ce sont des fournisseurs de lait à Montréal et éviter la critique $[\mathrm{sic}] \mathrm{.}^{78}$

Malgré les efforts des gouvernements et des vétérinaires pour accélérer l'examen des troupeaux, la critique ne pourra être évitée; elle prendra la forme d'un blocus de l'approvisionnement.

L'inspection des fermes et des laiteries et le test de dépistage de la tuberculose bovine imposés par la ville de Montréal sont des exemples de mesures réglementaires qui vont encadrer le marché du lait de consommation entre 1900 et 1930. Elles ne touchent pas directement les termes du commerce, mais modifient plutôt la manière

77 Lettre de J.-S. Jasmin au vétérinaire en chef Bédard, du service de l'élevage du ministère de l'Agriculture de la province de Québec, 25 avril 1927.

78 Ibid., 30 avril 1927. Parmi les noms soumis par le chef du service de l'élevage se trouvent quelques membres de la future APLPQ, notamment le directeur Ernest Chartrand. Déjà en 1927, Jasmin perçoit des signes d'impatience dans son secteur: «Je vous ferai remarquer qu'un certain groupe, je vous les nommerai au besoin, monte les esprits dans ce district, pour profiter de l'occasion pour critiquer le gouvernement.» Lettre du 19 décembre 1927. 
de produire le lait. Cependant, le contrôle par l'État du prix de vente du lait ne tardera pas à être instauré.

La crise économique fait accélérer l'afflux de fournisseurs vers les laiteries montréalaises: du coup, la situation de surapprovisionnement qui subsiste de façon latente depuis le milieu des années 1920 s'aggrave. La chute des prix et la transformation du mode de paiement du lait - un prix de base et un prix de surplus - qui en résultent défavorisent tous les fournisseurs, y compris les plus gros expéditeurs. L'état sérieux d'instabilité et de détérioration des conditions du marché du lait de consommation amène à partir de 1932 les gouvernements fédéral et provincial à enquêter sur la situation. À la suite de ces travaux, le ministère de l'Agriculture de la province institue la Commission de l'industrie laitière, dans le but précis de faire disparaître «...la véritable anarchie qui règne dans le commerce du lait» ${ }^{79}$. Les commissaires obtiennent le pouvoir de fixer, après audition des demandes des producteurs et des laiteries distributrices, les conditions de mise en marché du lait de consommation - soit essentiellement les prix de gros et de détail - pour les villes de la province. La toute première ordonnance, datée du 29 juin 1934, règle les termes de la vente sur le territoire du grand Montréal ${ }^{80}$.

Bien qu'il soit difficile d'évaluer la portée des décisions de cette commission, la détermination d'un prix plancher s'avère un important acquit pour les producteurs québécois de lait de consommation. La grande majorité des producteurs laitiers, ceux qui vendent aux fabriques, ne pourront se prévaloir d'un pareil contrôle du prix de vente pendant ces années de crise. Cette reconnaissance explicite de la division des marchés suivant l'usage du lait permet la consolidation de la position des fournisseurs de lait de consommation sur les marchés urbains. Incidemment, elle a suscité la création d'un rassemblement provincial des associations de ces producteurs spécialisés ${ }^{81}$. Cette dernière organisation deviendra, après la Seconde Guerre mondiale,

79 Selon les mots du ministre de l'Agriculture du moment, Adélard Godbout, cité dans James Iain Gow, Histoire de l'administration publique québécoise, 1867-1970 (Montréal, Université de Montréal/Institut d'administration publique du Canada, 1986), 89. Voir aussi Firmin Létourneau, «La politique agricole», Esdras Minville dir., L'agriculture (Montréal, Fides, 1943), 367.

80 Gazette officielle du Québec, 1934, 3009-3010. La commission poursuit ses activités en fixant les conditions de ventes pour les principales zones urbaines de la province.

81 La formation d'un premier regroupement provincial des producteurs de lait de consommation se produit pendant cette période (1934-1937). Certains procès-verbaux de cette association, les plus anciens datés de janvier 1937, sont conservés aux Archives nationales du Québec, centre de Québec, fonds Association des producteurs de lait (P425), articles 1 à 3. 
un puissant groupe de pression qui influencera - à son profit - les politiques relatives au commerce des produits laitiers ${ }^{82}$.

\section{CONCLUSION}

L'étude de la production et de la vente du lait de consommation entre 1900 et 1930 pose de sérieux problèmes de documentation: au contraire de la fabrication et de l'exportation de beurre et de fromage, ce commerce a été beaucoup moins surveillé par les autorités gouvernementales. J'ai essayé de pallier ces difficultés en rassemblant des éléments d'information provenant de sources diverses. Cet exposé milite en faveur de nouveaux travaux sur les modalités d'intégration des agriculteurs québécois aux divers marchés des denrées alimentaires pour la période qui précède la Seconde Guerre mondiale. Dans chaque cas, il importe de cerner les fluctuations des conjonctures internationale et régionale des marchés, de délimiter la structure et l'implantation des réseaux de transformation et de mise en marché, d'examiner le rôle des intermédiaires (agents d'exportation, experts gouvernementaux), d'évaluer finalement le degré de participation des producteurs agricoles. De plus, une attention toute particulière doit être portée à la genèse et à la mise en place de la réglementation qui fixe les conditions de production et de mise en marché.

Pareilles études pourraient - et devraient - être menées pour les principales productions commerciales. Je pense notamment à la vente de tabac, en raison de la place croissante que cette culture occupe dans le système de production des agriculteurs de la rive nord de la plaine de Montréal. Nous disposons sur ce sujet d'une documentation relativement abondante. Régi et taxé par le gouvernement fédéral, le commerce du tabac semble s'effectuer principalement avec les grandes compagnies de Montréal. Ici encore, les producteurs se sont organisés en coopérative, afin de partager les achats d'intrants, les frais de préparation des feuilles, et pour contrôler la mise en marché de leur production. Le commerce du foin, celui des œufs et de la volaille, les abattoirs régionaux et les conserveries méritent, eux aussi, une attention particulière. Ultimement, la synthèse de ces recherches sectorielles permettra de mieux comprendre les alternatives et les choix des producteurs dans cette première phase de commercialisation de l'agriculture familiale québécoise. 